\title{
Polarization of a stored beam by spin filtering
}

\author{
Christian Weidemann ${ }^{1,2, a}$ for the PAX collaboration \\ ${ }^{1}$ Dipartimento di Fisica, Università di Ferrara, I-44121, Ferrara, Italy \\ ${ }^{2}$ Forschungszentrum Jülich, Institut für Kernphysik, D-52425 Jülich, Germany
}

\begin{abstract}
In 2011 the PAX Collaboration has performed a successful spin-filtering test using protons at $T_{\mathrm{p}}=49.3 \mathrm{MeV}$ at the COSY ring, which confirms that spin filtering is a viable method to polarize a stored beam and that the present interpretation of the mechanism in terms of the proton-proton interaction is correct. The equipment and the procedures to produce stored polarized beams was successfully commissioned and are established. The outcome of the experiment is of utmost importance in view of the possible application of the method to polarize a beam of stored antiprotons.
\end{abstract}

\section{Introduction}

Polarized antiprotons allow unique access to a number of fundamental physics observables. One example is the transversity distribution which would be directly measurable via Drell-Yan production in double polarized antiproton-proton collisions (Fig. 1). This and other observables, which are accessible via $\vec{p} \vec{p}$ scattering experiments [1], led the Polarized Antiproton eXperiments (PAX) collaboration to propose such investigations at the High Energy Storage Ring (HESR) of the Facility for Antiproton and Ion Research (FAIR)[1].

Figure 1: The Drell-Yan process in protonantiproton interaction. A valence quark from the proton annihilates with a valence antiquark of the antiproton and the generated virtual photon eventually decays into a lepton-antilepton pair.

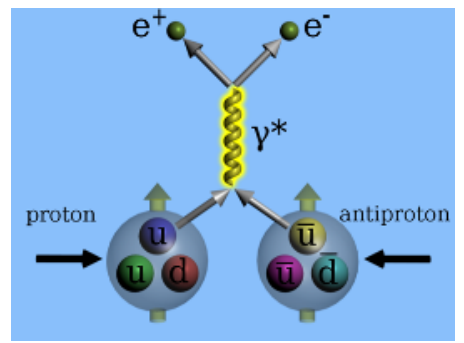

Polarizing an originally unpolarized beam of spin- ${ }^{1} / 2$ particles is feasible either by selectively reversing the spin of particles in one spin state ("spin flipping") or by selectively discarding particles in one spin state ("spin filtering") (Fig. 2). In a recent experiment performed at COSY in Jülich, however, the $e \vec{p}$ spin-flip cross sections were shown to be too small to allow for the efficient production of polarized antiprotons [2].

Spin filtering using the spin-dependent part of the nucleon-nucleon interaction is the only experimentally demonstrated viable method to polarize antiprotons. After the first experimental evidence with a $23 \mathrm{MeV}$ proton beam at the TSR ring in Heidelberg [3], an additional measurement, has been recently performed at the COSY storage ring to provide an independent confirmation of the method and of its present theoretical interpretation [4-6].

\footnotetext{
ae-mail: c.weidemann@fz-juelich.de
} 

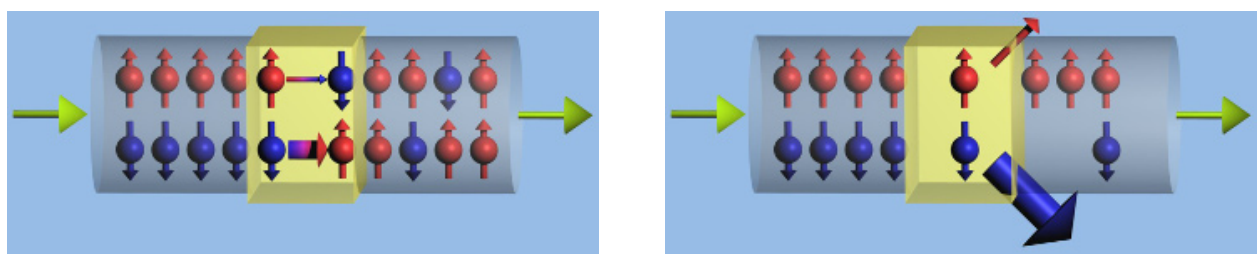

Figure 2: The two possible ways to polarize a beam of spin- $1 / 2$ particles in a storage ring are spin flipping (left panel) and spin filtering (right panel).

\section{Principle of measurement}

The spin-filtering method is based on the spin-dependent part $\sigma_{1}$ of the total hadronic cross section [7],

$$
\sigma_{\text {tot }}=\sigma_{0} \pm \sigma_{1} \cdot Q
$$

where $\sigma_{0}$ is the spin-independent part and $Q$ is the nuclear polarization of the target. The positive (negative) sign denotes parallel (antiparallel) orientation of the spins of beam and target protons. As a consequence of the interaction, the intensity of the spin-up $\left(N^{\uparrow}\right)$ and spin-down $\left(N^{\downarrow}\right)$ protons in the ring decreases exponentially with different time constants leading to a polarization buildup as function of time

$$
P(t)=\frac{N^{\uparrow}(t)-N^{\downarrow}(t)}{N^{\uparrow}(t)+N^{\downarrow}(t)}=\tanh \left(\frac{t}{\tau_{1}}\right) .
$$

The spin-dependent effective polarization buildup cross section $\tilde{\sigma}_{1}$ can be extracted from the observed time constant $\tau_{1}$ of the buildup rate via

$$
\frac{d P}{d t} \approx \frac{1}{\tau_{1}}=\tilde{\sigma}_{1} Q d_{\mathrm{t}} f
$$

where $d_{\mathrm{t}}$ is the target areal density in atoms $/ \mathrm{cm}^{2}, f$ the particle revolution frequency [8]. $\tilde{\sigma}_{1}$ accounts for the fact that only protons scattered at angles larger than the acceptance angle of the storage ring $\Theta_{\text {acc }}$ contribute to the spin-filtering process $\tilde{\sigma}_{1}=\sigma_{1}\left(\Theta>\Theta_{\text {acc }}\right)$.

\section{Experimental setup}

The COSY accelerator and storage ring with the installations utilized in the investigation is shown in Fig. 3. The two main components of the experimental apparatus used for the measurement are the polarized hydrogen gas target $[9,10]$ making use of a storage cell, installed at the PAX interaction point, and the beam polarimeter. Beam polarimetry has been accomplished by detecting elastically scattered protons off a deuterium cluster target [11] by means of two silicon tracking telescopes [12]. By means of an intensive program of machine development studies (described in [13]) a beam lifetime of $8000 \mathrm{~s}$ could be achieved at beam energy $T_{\mathrm{p}}=49.3 \mathrm{MeV}$. Phase space cooling of the stored beam is achieved by the electron cooler [14]. The use of a spin-flipper allows to reverse the vertical polarization of the beam.

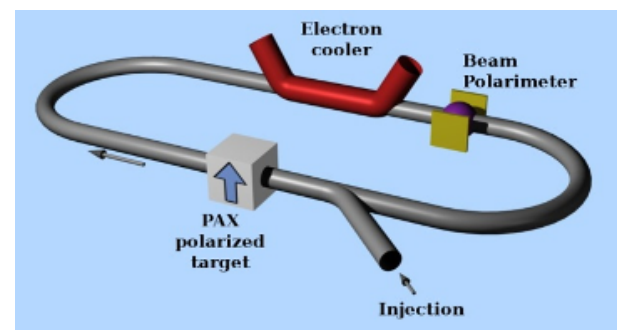

Figure 3: The COSY storage ring. The polarized hydrogen target is installed in one of the straight sections of the ring. The electron cooler followed by the beam polarimeter are installed in the opposite straight section. 


\section{Spin filtering cycle}

To perform the spin-filtering experiment, a dedicated spin-filtering cycle has been implemented according to the following scheme:

- An unpolarized proton beam is injected in the COSY ring at a beam energy of $45 \mathrm{MeV}$. The beam is cooled and subsequently accelerated to $49.3 \mathrm{MeV}$. This energy has been chosen for the spin-filtering experiments, because of existing data of the analyzing power in proton-deuteron elastic scattering.

- At this point the spin-filtering starts. Polarized hydrogen is injected into the storage cell at the PAX interaction point. The holding field coils, orienting the target polarization, are powered on in either up or down orientation for the duration of the spin-filtering period. Two different spin-filtering periods have been adopted: one lasting for $12000 \mathrm{~s}$, and a longer one of $16000 \mathrm{~s}$, corresponding to about 1.5 and 2 times the measured beam lifetime ( $8000 \mathrm{~s})$.

- At the end of the spin-filtering period, the PAX polarized target is switched off, the ANKE deuterium-cluster target is switched on and the data acquisition of the beam polarimeter starts. The beam polarization is determined by measuring the left-right asymmetry in $\vec{p} d$ elastic scattering as explained in [15]. Reversing the beam polarization during this period allows for the determination of the induced beam polarization within each cycle, thereby reducing systematic errors.

\section{Results}

The spin-filtering cycles have been repeated for the up and down directions of the target holding field. The beam polarization obtained from spin-filtering cycles of different length for the two target spinorientations is presented in Fig. 4 (left panel). In order to investigate fake asymmetries as possible sources of systematic errors, the same analysis procedure has been applied to the zero measurement (filter time $=0 \mathrm{~s}$ ), where no spin-filtering took place and no polarization was induced in the beam. This measurement can be interpreted as a determination of the systematic error of the beam polarization. A linear fit to the five points shown in Fig. 4 allows us to determine $\frac{d P}{d t}=(4.8 \pm 0.8) \cdot 10^{-7} \mathrm{~s}^{-1}$. By making use of the measured polarization buildup and additional information like target polarization and density, and beam revolution frequency, the effective polarizing cross section has been derived [8]:

$$
\tilde{\sigma}_{1}^{\text {meas }}=-23.4 \pm 3.9 \text { (stat.) } \pm 1.9 \text { (syst.) } \mathrm{mb}
$$

The spin-dependent cross-section measured at COSY is presented in Fig. 4 (right panel) together with the other existing measurement performed by the FILTEX collaboration $[3,16]$. The solid line represents the theoretical prediction from the nucleon-nucleon interaction. The good agreement between measurement and theory confirms that spin-filtering of a stored proton beam is well-described taking into account the contributions from proton-proton scattering.

\section{Summary}

The PAX Collaboration has successfully completed a spin-filtering experiment using a beam of protons at COSY. The measurement confirms that spin-filtering can be effectively used to polarize a stored beam in situ and that our understanding of the mechanism in terms of the proton-proton interaction is correct. The achievement is of fundamental importance in view of the possible application of the method to polarize a beam of stored antiprotons. In this respect, the existing theoretical predictions for the polarization buildup with antiprotons are affected by the lack of knowledge of the proton-antiproton interactions. For this reason, a direct measurement of the polarizing cross sections in proton-antiproton interactions constitutes an inevitable step towards the design of a dedicated polarizer ring. 

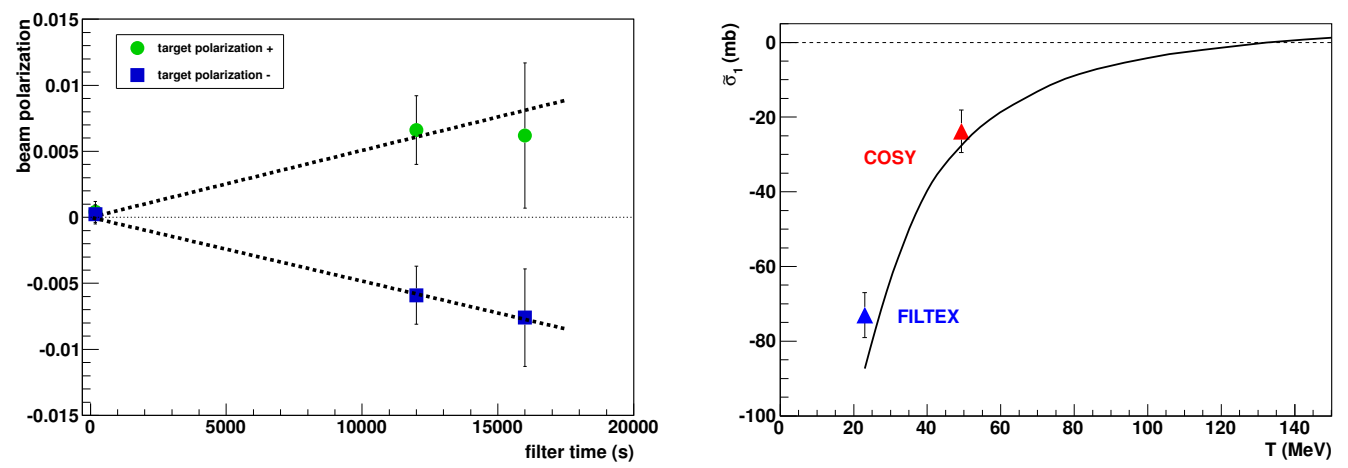

Figure 4: Left panel: Polarization induced in the beam after filtering for different times and different signs of the target polarization. The induced polarization in the beam has the same sign as the target polarization, reflecting the negative sign of the polarizing cross section. A linear fit (dashed lines) to the five points yields $\frac{d P}{d t}$. Right panel: Measured spin-dependent polarizing cross section for the interaction (only statistical errors are shown). The solid line represents the prediction from the SAID database [17]. The prediction are basically independent from the ring-acceptance in the interval of values interested by the TSR and COSY rings.

\section{References}

[1] P. Lenisa and F. Rathmann for the PAX collaboration, Technical Proposal for AntiprotonProton Scattering Experiments with Polarization (2006); available from the PAX website: http://collaborations.fz-juelich.de/ikp/pax/ .

[2] D. Oellers et al., Phys. Lett. B674, 269 (2009), arXiv:nucl-ex/0902.1423.

[3] F. Rathmann et al., Phys. Rev. Lett. 71, 1379 (1993).

[4] A. Milstein, V. Strakhovenko, Phys. Rev. E72, 066503 (2005).

[5] N. Nikolaev, F. Pavlov, in: Proceedings of the 17th International Spin Physics Symposium (SPIN2006), Kyoto, 2006, arXiv:hep-ph/0601184.

[6] N.H. Buttimore, D. O’Brien, Eur. Phys. J. A35, 47 (2008).

[7] J. Bystricky, F. Lehar, and P. Winternitz, J. Phys. (France) 39, 1 (1978).

[8] W. Augustyniak et al., Phys. Lett. B 718, 64 (2012).

[9] A. Nass, et al., Nucl. Instr. Meth. A 505, 633 (2003).

[10] C. Baumgarten et al., Nucl. Instrum. Meth. A 482, 606 (2002).

[11] A. Khoukaz, et al., Eur. Phys. J. D 5, 275 (1999).

[12] R. Schleichert, et al., IEEE Trans. Nucl. Sci. 50, 301 (2003).

[13] C. Weidemann, Ph.D. thesis, University Cologne (2011) available from http://collaborations.fzjuelich.de/ikp/pax/public_files/theses/thesisCh.Weidemann.pdf;

C. Weidemann et al., Machine development for spin-filtering studies at COSY, in preparation

[14] H. Stein et al., (2011), arXiv:physics.acc-ph/1101.5963.

[15] D. Oellers, Ph.D. thesis, University Cologne (2010) available from http://collaborations.fzjuelich.de/ikp/pax/public_files/theses/thesisD.Oellers.pdf

[16] F. Rathmann, Ph.D. thesis, Phillips-Universität Marburg, January 1994

[17] SAID, Nucleon-nucleon scattering database, Center for Nuclear Studies, Department of Physics, George Washington University, USA. 